\title{
Estudio inicial de un chatbot para estudiantes de la modalidad virtual de la Escuela Interamericana de Bibliotecología
}

\author{
María Teresa Múnera Torres* \\ Leidy Marisol Salazar Álvarez* \\ Arbey Stiven Osorio 0sorio*
}

\begin{abstract}
Artículo recibido:
28 de abril de 2021

Artículo aceptado:

1 de septiembre de 2021

Artículo de investigación
\end{abstract}

\section{Resumen}

La inclusión de las TIC en la educación ha generado diversos retos, entre ellos, el imaginario tergiversado de lo que implica y significa la modalidad virtual, el uso de las plataformas y la apropiación de los procesos logísticos, académicos y administrativos. En este contexto, surgen diversas preguntas por parte de los estudiantes que hacen imperioso brindar respuestas claras y oportunas. Aun más cuando estos estudiantes son campo de estudio de la información, quienes reconocen que la transferencia de la información contribuye en la optimización de los procesos de comunicación y de manera específica en los entornos de formación. Es así como el

* Escuela Interamericana de Bibliotecología, Universidad de Antioquia, Colombia

maria.munera@udea.edu.co marisol.salazar@udea.edu.co arbey.osorio@udea.edu.co

INVESTIGACIÓN BIBLIOTECOLÓGICA, vol. 36, núm. 90, enero/marzo, 2022, México, ISSN: 2448-8321 pp. 13-30 
presente artículo dará cuenta de una experiencia investigativa que tuvo como objetivo principal diseñar una propuesta de asistente virtual que permitiera dar solución a las dudas de los estudiantes de archivística y bibliotecología de la modalidad virtual de la Escuela Interamericana de Bibliotecología -EIB- de la Universidad de Antioquia, con el fin de optimizar los procesos comunicativos entre los estudiantes y quienes coordinan las dinámicas de virtualidad en la institución. Dicho proceso de investigación se fundamentó en la metodología design thinking, que favoreció un estado de empatía con la comunidad académica y posibilitó la identificación de las inquietudes más recurrentes de los estudiantes y sus respectivas categorías temáticas principales. En este sentido, este fue un estudio inicial de carácter exploratorio y descriptivo que logró obtener información que servirá de insumo para una próxima fase de desarrollo de un asistente virtual para la EIB.

Palabras clave: Chatbot; Asistente Virtual; Educación Virtual; Escuela Interamericana de Bibliotecología; Universidad de Antioquia

Initial study of a chatbot for virtual modality students from the Inter-American School of Librarianship María Teresa Múnera Torres, Leidy Marisol Salazar Álvarez and Arbey Stiven Osorio Osorio

\section{Abstract}

The inclusion of ICT (TIC for the Spanish acronyms) in education has generated various challenges, among them the distorted imagery of what the virtual modality implies and means; the use of platforms and the appropriation of logistical, academic and administrative processes. In this context, various questions arise from students who think it is an imperative to provide clear answers timely. Even more so, when these students belong to the field of study of information and recognize that the transfer of information contributes to the optimization of communication processes, specifically in training environments. This is why this article will report on a research experience whose main objective was to design a proposal for a virtual assistant that allowed solving the doubts of archival and library 
students in the virtual modality of the Inter-American School of Library Science -EIB- of the University of Antioquia, in order to optimize communication processes between students and those who coordinate the dynamics of virtuality in the institution. Such research process was based on the design thinking methodology that favored a state of empathy with the academic community which made it possible to identify the most recurrent concerns of students and their respective main thematic categories. In this sense, this was an initial exploratory and descriptive study that managed to obtain information which will serve as input for a next phase of development of a virtual assistant for the EIB.

Keywords: Chatbot; Virtual Assistant; Virtual Education; Inter-American School of Librarianship; University of Antioquia

\section{INTRODUCCIÓN}

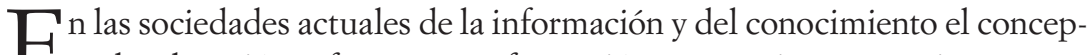
- to de educación sufre una transformación y se empieza a asumir como un acto relacional, donde se establecen vínculos basados en la comunicación e interacción de profesores y estudiantes, y de estudiantes con estudiantes. Estas interacciones han obligado a asumir las TIC como un aliado fundamental para el desarrollo y la implementación de dichos procesos educativos.

En este contexto, los asistentes virtuales o chatbots tienen un potencial educativo importante, precisamente por su capacidad comunicativa mediante lenguaje natural y porque aportan elementos de interactividad, aunque es importante destacar que estos asistentes no sustituyen a las personas, pues su función es complementaria; por lo tanto, el trabajo de los docentes y del personal administrativo de una institución sigue siendo vital.

Es por esto que investigadores, desarrolladores y docentes se han puesto a la tarea de trabajar conjuntamente con la intención de estudiar, diseñar e implementar asistentes virtuales que respondan a las necesidades de información del estudiantado. Así lo demuestran los trabajos de Ch'ng, Yeong y Ang (2019), Almurtadha (2019), Sinha et al. (2020), y Jo et al. (2020), donde se hace énfasis en el análisis contextual de la información que se obtiene en espacios como foros, bases de datos y aulas educativas virtuales, lo que les permite caracterizar las necesidades informacionales de sus públicos objetivos y, con dicha caracterización, desarrollar asistentes virtuales que respondan a 
los requerimientos contextuales de un grupo específico, lo que disminuye el tiempo de espera para una respuesta, brinda una orientación más acertada y contextualizada, mejora la predictibilidad y número de aciertos a las soluciones que brindan los chatbots, y facilita la caracterización de las necesidades de los usuarios y su comportamiento lingüístico en espacios virtuales.

Dentro del contexto de los nuevos paradigmas de formación archivística y bibliotecológica, resulta muy pertinente incluir dinámicas que orienten a los futuros profesionales de estas áreas del conocimiento en el uso, diseño y aplicación de asistentes virtuales que contribuyan con la optimización de los procesos comunicativos dentro de su formación, y a futuro en las diferentes de unidades de información a las que ingresen a laborar.

Precisamente, sirviéndose de las posibilidades de los chatbots, esta investigación tiene como objetivo principal diseñar una propuesta de asistente virtual que permita dar solución a las dudas de los estudiantes de la modalidad virtual de la Escuela Interamericana de Bibliotecología (EIB) de la Universidad de Antioquia, con el objetivo de optimizar los procesos comunicativos entre los estudiantes y quienes coordinan las actividades de modalidad virtual en la institución.

La investigación surge en respuesta a la inconformidad que varios estudiantes manifestaron con respecto a la modalidad virtual y su oferta dentro de la Escuela, lo que evidencia la necesidad de disponer de mecanismos de información que aclaren las dudas de los estudiantes sobre este proceso. En tal sentido, un asistente virtual o chatbot se puede convertir en un mecanismo para favorecer la comunicación entre los estudiantes y quienes lideran los procesos de educación virtual, y propiciar el cambio del imaginario tergiversado sobre lo que significa esta modalidad, su planeación, sus procesos y su oferta.

Para llevar a cabo esta investigación se plantearon los siguientes objetivos específicos: a) Identificar los componentes y características que debe tener un asistente virtual para la optimización de los procesos de comunicación entre estudiantes y quienes coordinan los procesos de virtualidad; b) Detectar los problemas e inquietudes más recurrentes en los estudiantes de la modalidad virtual de la EIB, y c) Definir términos de la educación virtual que debe reconocer un asistente virtual, a fin de apoyar los procesos comunicativos entre estudiantes y personal administrativo. En este sentido, este artículo pretende brindar un panorama sobre el valor de los asistentes virtuales en el mejoramiento de los procesos de comunicación en diferentes contextos y, de manera especial, en el de la educación superior.

A continuación, se presenta la metodología desarrollada, basada en el design thinking, conformada por las etapas siguientes: empatizar, definir, idear, prototipar y evaluar, las cuales incluyen a su vez una serie de técnicas e instrumentos 
metodológicos que contribuyeron con el desarrollo de la investigación. Posteriormente, se presentan algunos de los resultados logrados que evidencian la existencia de categorías temáticas relacionadas con las dudas principales que manifiestan los estudiantes de la modalidad virtual de la EIB. Estos resultados son puestos a discusión con algunos referentes para, finalmente, culminar con las conclusiones de la dinámica investigativa y la bibliografía correspondiente.

Antes de continuar, es importante mencionar que este proyecto de investigación fue inscrito en el Centro de Investigaciones en Ciencia de la Información (CICINF), dependencia de la Escuela Interamericana de Bibliotecología (EIB) de la Universidad de Antioquia, y financiado por la convocatoria Con TIC Investigo de la Vicerrectoría de Docencia de la misma universidad.

\section{Metodología}

Para el desarrollo de esta investigación se empleó un enfoque cualitativo, de alcance exploratorio y descriptivo, con el fin de analizar e interpretar los hechos de manera dinámica. Asimismo, en esta etapa inicial se recogieron los insumos necesarios para una nueva investigación que se convierta en una segunda etapa del proyecto, en la cual se logre el desarrollo y la disposición de un asistente virtual para los estudiantes de la EIB que contribuya con el mejoramiento de los procesos de comunicación de la institución.

Este trabajo se apoyó en la metodología design thinking, la cual se fundamenta en un proceso social y comunicativo dividido en cinco etapas (Plattner, 2018):

a. Empatizar: se centra en entender a las personas y sus necesidades mediante la observación, la escucha y la empatía.

b. Definir: consiste en delimitar el foco de acción, definir y replantear los conceptos, basándose en el proceso de empatía con los usuarios y su contexto.

c. Idear: en esta etapa se empiezan a crear soluciones innovadoras para los problemas o focos de acción definidos, se construyen conceptos y se detallan los recursos necesarios para hacer prototipos.

d. Prototipar: se enfoca en aterrizar las ideas al crear elementos informativos como dibujos, artefactos y objetos que se acerquen o ilustren la solución final, mostrando sus características funcionales, formales y de uso.

e. Evaluar: consiste en validar el prototipo y empatizar nuevamente con los usuarios, quienes encaminarán la posterior toma de decisiones estratégicas dependiendo de su validación. 
En esta línea, la metodología design thinking busca una comprensión profunda de las necesidades de los usuarios y, en el caso específico de la presente investigación, ayudó a comprender las necesidades de información de los estudiantes de archivística y bibliotecología de la modalidad virtual de la EIB. En la Tabla 1 se pueden apreciar, por cada una de las etapas, las técnicas e instrumentos empleados en la recolección y análisis de información.

\begin{tabular}{|l|l|l|l|}
\hline \multicolumn{2}{|c|}{ Etapa } & \multicolumn{1}{c|}{ Técnicas } & \multicolumn{1}{c|}{ Instrumentos } \\
\hline 1 & Empatizar & $\begin{array}{l}\text { Búsqueda documental } \\
\text { Análisis de texto } \\
\text { Grupo focal } \\
\text { Entrevistas } \\
\text { Encuesta }\end{array}$ & $\begin{array}{l}\text { Ficha documental } \\
\text { Matriz de datos y categorías } \\
\text { Guía de grupo focal } \\
\text { Guías de entrevistas } \\
\text { Cuestionario }\end{array}$ \\
\hline 2 & Definir & Mapas mentales & Organizador gráfico \\
\hline 3 & Idear & Brainstorming & Guía de brainstorming \\
\hline 4 & Prototipar & Plano de producto/servicio & Tablero de dibujo \\
\hline 5 & Evaluar & Grupo focal & Guía de grupo focal \\
\hline
\end{tabular}

Tabla 1. Etapas, técnicas e instrumentos aplicados en la investigación

Fuente: elaboración propia

Es importante destacar la etapa empatizar, ya que es determinante al definir las necesidades de información de los estudiantes. En ella se optó por realizar entrevistas con la jefatura académica y las coordinaciones de Bienestar y Virtualidad; de igual forma, se realizaron grupos focales con los monitores (estudiantes que cumplen funciones auxiliares para orientar y acompañar a profesores y a estudiantes de la modalidad virtual), el equipo de soporte que atiende y soluciona los problemas técnicos relacionados con la plataforma educativa, los estudiantes y profesores de la modalidad virtual de la EIB.

Estas entrevistas y grupos focales facilitaron la construcción de una encuesta que fue aplicada a los estudiantes de la modalidad virtual. Posteriormente, se analizaron los foros de preguntas extraídos de la plataforma educativa. El análisis de la encuesta y de los foros permitió, en la segunda etapa, definir las categorías temáticas de las preguntas más recurrentes que presentaban los estudiantes, las cuales fueron esquematizadas a partir de organizadores gráficos; los mapas generados fueron un insumo importante en la tercera etapa para identificar e idear las características necesarias en el diseño de un asistente virtual. Dichas características y categorías temáticas fueron elementos vitales en la etapa prototipar, al favorecer la creación de una arquitectura de software para un posible chatbot. 
Finalmente, en la etapa evaluar se realizó un grupo focal con estudiantes y profesores, a quienes se les solicitó diligenciar una encuesta de percepción sobre la propuesta de chatbot diseñada. La relación entre los públicos y las técnicas aplicadas se ilustran en la Tabla 2.

\begin{tabular}{|l|l|}
\hline \multicolumn{1}{|c|}{ Entrevistas } & \multicolumn{1}{c|}{ Grupos focales } \\
\hline $\begin{array}{l}\text { Coordinación de Bienestar } \\
\text { Coordinación de Virtualidad } \\
\text { Jefatura Académica }\end{array}$ & $\begin{array}{l}\text { Monitores } \\
\text { Equipo de soporte de la unidad de virtualidad } \\
\text { Estudiantes } \\
\text { Profesores }\end{array}$ \\
\hline \multicolumn{1}{|c|}{ Encuesta } & \multicolumn{1}{c|}{ Análisis de texto } \\
\hline $\begin{array}{l}\text { Inquietudes de los estudiantes de la } \\
\text { modalidad virtual. } \\
\begin{array}{l}\text { Percepción del proyecto por parte de } \\
\text { estudiantes y profesores de la modalidad } \\
\text { virtual. }\end{array}\end{array}$ & $\begin{array}{l}\text { Participación de estudiantes en foros de preguntas } \\
\text { alojados en las aulas virtuales. }\end{array}$ \\
\hline
\end{tabular}

Tabla 2. Públicos y técnicas aplicadas

Fuente: elaboración propia

Las actividades presentadas en la Tabla 2 reflejan el reconocimiento de diversos actores que intervienen en los procesos de modalidad virtual en la EIB y la dinámica social de base necesaria para desarrollar el esquema de flujo de información oportuno y, con ello, crear un arquetipo de asistente virtual apropiado para el público objetivo y el contexto que lo rodea.

\section{ANÁlisis y RESUltados}

La dinámica inicial que se contempló en el desarrollo de la investigación se basó en la búsqueda bibliográfica con el propósito de identificar los componentes y las características que debe tener un chatbot, lo cual implicó, además, la revisión de experiencias de aplicación de asistentes virtuales en diferentes tipos de instituciones. Para ello se realizó un rastreo de términos relacionados con el ámbito de los asistentes virtuales, a fin de conformar una base de datos terminológicos en este campo específico del saber para que a su vez sirviera de complemento e insumo para el estudio inicial de la propuesta del chatbot de la EIB.

Por otra parte, el enfoque cualitativo favoreció el reconocimiento de los actores involucrados en los procesos de educación virtual de la EIB, los cuales fueron indispensables para generar un estado de empatía en el equipo de 
investigación. Este sentir fue necesario para identificar, de forma oportuna, las necesidades de información de los estudiantes y las categorías temáticas principales asociadas a las preguntas más frecuentes que se pueden presentar.

En el semestre 2020/1 la EIB contaba con un total de 456 estudiantes, $65 \%$ de los cuales estaban matriculados, por lo menos, en un (1) curso de la modalidad virtual; $19 \%$ de ellos se encontraba asociado al programa de Bibliotecología, $81 \%$ se encontraba matriculado en cursos del programa de Archivística y, entre éstos, $13 \%$ de los estudiantes tenían asignaturas compartidas entre ambos programas. Estos datos pueden apreciarse en la Figura 1.
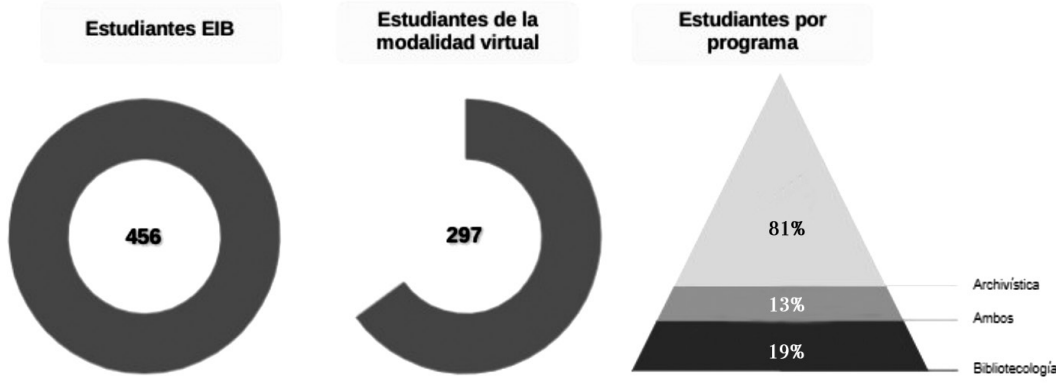

Figura 1. Población del objeto de estudio Fuente: elaboración propia

Es evidente que hay mayor cantidad de estudiantes del programa de Archivística, ante esto es conveniente mencionar que en 2018 la Escuela empezó a ofrecer a sus egresados y estudiantes de la Tecnología en Archivística un ciclo profesional con asignaturas virtualizadas para los dos últimos niveles (séptimo y octavo), por tanto, el público objetivo de los cursos de la modalidad virtual fueron, inicialmente, personas interesadas en concluir su formación como profesionales en el programa de Archivística. La mayoría de estas personas se encuentran en regiones distantes de la Ciudad Universitaria (ubicada en Medellín) y se ven altamente beneficiadas con esta modalidad.

Por otra parte, desde 2019 se han ofrecido para el programa de Bibliotecología algunos cursos (la mayoría electivos) en modalidad virtual y aulas de apoyo a las actividades de la presencialidad; es decir, que la Escuela ofrece algunas asignaturas en ambas modalidades: presencial y virtual. Aunque en principio se buscaba llegar prioritariamente a las regiones de difícil acceso, algunos estudiantes cercanos a la Ciudad Universitaria también han matriculado asignaturas en modalidad virtual. 
Con el fin de conocer el proceso de modalidad virtual llevado a cabo en la EIB e identificar las inquietudes más frecuentes de los estudiantes se realizaron tres entrevistas (con la Jefatura Académica y con las coordinaciones de Bienestar y Virtualidad), cuatro grupos focales (con el equipo de soporte de la plataforma educativa, los monitores de los programas virtuales de la EIB, estudiantes y profesores de la modalidad virtual), y el análisis de los foros de preguntas de 14 cursos que se ofrecen en dicha modalidad.

Las entrevistas realizadas y los grupos focales facilitaron la creación de una encuesta para identificar los problemas e inquietudes más recurrentes de los estudiantes. Se recibieron 41 formularios diligenciados, que equivalen a $14 \%$ de estudiantes de la modalidad virtual. Si bien esta tasa de retorno no fue la esperada, es conveniente aclarar que la encuesta fue aplicada en el marco de la pandemia generada por la COVID-19, la cual ha propiciado cambios en las diversas dinámicas y actividades sociales; por tanto, era de esperarse que los estudiantes estuvieran saturados de información y de actividades digitales, lo que posiblemente limitó el diligenciamiento e interés en responder la encuesta que no se podía postergar, debido a los compromisos asumidos según el cronograma de investigación. No obstante, los resultados se consideran de gran valor para el proceso de investigación y un insumo esencial para generar el flujo de diálogo y el estudio inicial de un asistente virtual.

De las 41 respuestas, 20 fueron diligenciadas por estudiantes del programa de Bibliotecología y 21 del programa de Archivística. La mayoría de los estudiantes $(66 \%)$ manifestó tener matriculados entre tres y cinco cursos en modalidad virtual. A continuación, se presentan los resultados más importantes de esta encuesta.

Se pudo observar que $63 \%$ de las respuestas pertenecen a estudiantes del género femenino (26). Por otra parte, la mayoría de encuestados se ubican en el rango entre 21 y 25 años (34 \%), seguido del rango 31 a 35 años (17 \%).

Adicionalmente, quienes más se interesaron en responder la encuesta fueron los estudiantes ubicados en los niveles académicos uno (20\%), tres (22\%) y seis ( $15 \%$ ); es relevante que muchos de los estudiantes del primer nivel sean de los más interesados en responder la encuesta. Asimismo, hubo mayor participación de los estudiantes ubicados en la ciudad de Medellín; si bien en esta ciudad está la sede central, llama la atención la falta de estudiantes de otros municipios y regiones, pues en principio la modalidad virtual en la EIB se propuso como una oportunidad para estudiantes de las zonas apartadas a la ciudad universitaria.

Por otra parte, se encontró que las principales instancias a las cuales se dirigen con mayor frecuencia los estudiantes fueron profesores de los cursos (35\%), Jefatura Académica (17 \%), Coordinación de Bienestar (13\%) y 
Coordinación de Virtualidad (12 \%). Asimismo, 32 \% de los estudiantes manifestó que presenta mayores inquietudes durante el desarrollo del semestre académico (Figura 2).

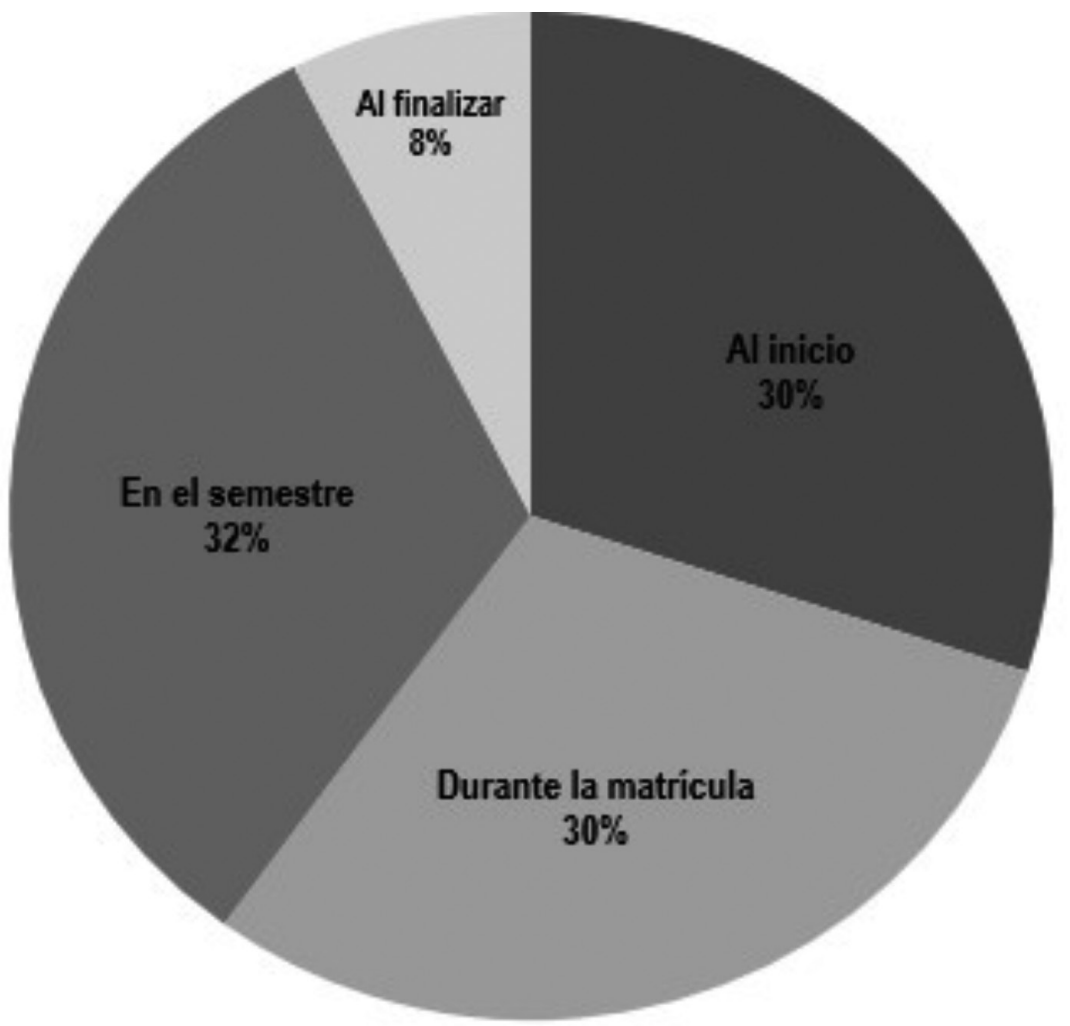

Figura 2. Momentos de dudas

Fuente: elaboración propia

Por otro lado, los medios de comunicación más utilizados para enviar sus inquietudes fueron el correo electrónico (98\% del total de respuestas) y WhatsApp (56\% del total de respuestas). Otros medios de comunicación menos empleados por los estudiantes fueron las llamadas telefónicas, la mensajería y los foros de la plataforma educativa, el diálogo presencial y las redes sociales. Con base en la información anterior, se evidencia en el WhatsApp una posibilidad para crear propuestas de implementación del chatbot con la API oficial de WhatsApp Business.

En cuanto a las categorías temáticas se identificó que las dudas más recurrentes han sido de orden académico $(17 \%)$, relacionadas principalmente con los cursos, sus profesores, su intensidad horaria y sus calificaciones; de 
carácter administrativo (17\%), donde se encontraron inquietudes en lo referente a procesos de matrícula, cancelaciones de cursos, grados, entre otros, y relacionadas con Bienestar Universitario (14\%), donde se observaron preguntas con relación a los apoyos sociales, el aprovechamiento del tiempo libre y los servicios ofrecidos por la Coordinación de Bienestar. Lo anterior se ilustra en la Figura 3.

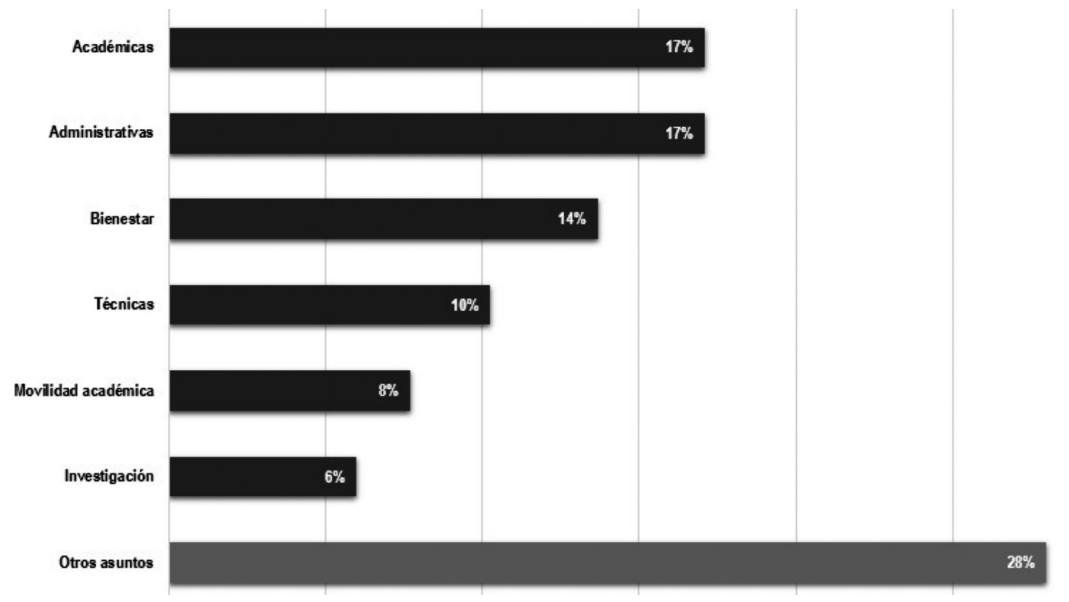

Figura 3. Categorías temáticas extraídas del análisis de preguntas frecuentes

Fuente: elaboración propia

Es importante aclarar que se encontraron inquietudes sobre otros aspectos y dependencias que no son gestionadas directamente por la EIB, entre las que se mencionan los servicios del Sistema de Bibliotecas, el Programa Institucional de Inglés y el Sistema de Estímulos Académicos; inquietudes que se ubicaron en la categoría "Otros asuntos".

Luego de indagar en el periodo académico 2020/1 con respecto a la participación de los estudiantes en los foros de preguntas correspondientes a 14 cursos alojados en la plataforma educativa, se encontró que 20 de ellos participaron por lo menos una vez en algún tipo de foro, sumando un total de 328 participaciones: 41 estaban relacionadas con foros de preguntas, en los cuales se logró apreciar que el mayor número (39\%) correspondió a dudas sobre actividades académicas, seguido de solicitudes de asesoría (10 \%), ampliación de fechas de actividades (10 \%), creación de grupos (10 \%), inquietudes sobre la grabación de los encuentros sincrónicos (10\%), inquietudes sobre materiales $(7 \%)$ y dificultades técnicas $(5 \%)$.

Con base en la información obtenida en las entrevistas, los grupos focales, las encuestas y en el análisis de los foros, se logró obtener el insumo, descrito 
en la Tabla 3, mediante el cual se precisan las características preliminares con las que debe contar un asistente virtual para la EIB.

\begin{tabular}{|l|l|}
\hline \multicolumn{1}{|c|}{ Característica } & \multicolumn{1}{c|}{ Descripción } \\
\hline Idioma & Español. \\
\hline Interoperabilidad & Web y móvil (a través de WhatsApp). \\
\hline Estilo conversacional & $\begin{array}{l}\text { Directo, con empleo de frases cortas y simples, sin tecnicismo } \\
\text { y con reconocimiento de siglas. }\end{array}$ \\
\hline Modelo & $\begin{array}{l}\text { Híbrido (con opciones predeterminadas, pero con la posibili- } \\
\text { dad de preguntar). }\end{array}$ \\
\hline Flujo de información & $\begin{array}{l}\text { Basado en las categorías temáticas principales encontradas } \\
\text { al momento de analizar los resultados de la aplicación de los } \\
\text { instrumentos de investigación. }\end{array}$ \\
\hline Personalidad & $\begin{array}{l}\text { Robot, joven, estudiante de la EIB, empático, propositivo, } \\
\text { paciente y simpático. }\end{array}$ \\
\hline Capacidad y forma de diálogo & $\begin{array}{l}\text { Comunicación textual basada en FAQ (preguntas frecuentes) } \\
\text { que requiere baja inteligencia artificial. }\end{array}$ \\
\hline
\end{tabular}

Tabla 3. Características preliminares para el asistente virtual de la EIB Fuente: elaboración propia

Teniendo en cuenta que esto es un estudio inicial con los resultados de investigación, se espera, en una segunda etapa, continuar con el desarrollo de la arquitectura de software que servirá de fundamento en el diseño y aplicación del asistente para la EIB, y permitirá dar solución a las dudas de los estudiantes de la modalidad virtual con el fin de optimizar los procesos comunicativos entre ellos y quienes coordinan las actividades de dicha modalidad en la institución.

\section{Discusión}

La palabra chatbot es una combinación entre dos términos en inglés: chat y bot. Chat significa conversar y se asocia con la comunicación en tiempo real que se realiza entre varias personas mediante un programa de internet; bot, por su parte, se relaciona con un robot o un programa informático que ejecuta automáticamente tareas repetitivas.

Para Abdul-Kader y Woods, "los asistentes conversacionales o mejor conocidos como bot o chatbot o chatterbot, al que se puede definir como un 
asistente virtual, es un conjunto de programas informáticos que poseen la habilidad de mantener una conversación con un ser humano mediante el lenguaje natural" (citado por Medrano, Tejerina, y Castillo, 2019: 3). Igualmente, y en palabras de Griol et al. (2011: 20), "un agente conversacional puede entenderse como un sistema automático capaz de emular a un ser humano en un diálogo con otra persona, con el objetivo de que el sistema suministre cierta información o lleve a cabo una determinada tarea”.

Para Holtgraves y Han (2007), la comunicación mediada por estas máquinas requiere comprender aspectos sociales y cognitivos propios del proceso comunicativo del humano con la máquina, y pese a que en la mayoría de ocasiones los bots generalizan las conversaciones, son cada vez más habituales y se involucran en aspectos sociales y cognitivos de la comunicación.

$\mathrm{Y}$ es que, para comprender el significado de un asistente virtual, es conveniente remontarse a la década de los años sesenta del siglo XX con el surgimiento de Eliza, una máquina con "inteligencia” que simulaba una terapeuta y reproducía conversaciones entre un psicoanalista y un paciente. En dicha época, Eliza tenía limitantes en su capacidad de memoria, base de datos y estructura de conocimiento (García Brustenga, Fuertes-Alpiste y Molas-Castells, 2018).

En la actualidad existen otras máquinas conocidas como Watson, Siri, Google New, Alexa y Cortana. Particularmente, el asistente IBM Watson fue desarrollado en 2006 para competir en un programa de televisión y desde entonces ha empleado el procesamiento de lenguaje natural y el aprendizaje automático para analizar grandes cantidades de datos.

Por su parte, Siri se presentó como una aplicación para iPhone que fue comprada por Apple para integrarla en 2011 como asistente de voz en el iPhone 4S. Por el contrario, Google Now fue desarrollado por Google y creado especialmente para el motor de búsqueda de Google en teléfonos móviles. De igual forma, Amazon desarrolló a Alexa en 2015 para emplearla en los altavoces inteligentes para el hogar. Uniéndose a este boom de los asistentes virtuales, Microsoft desarrolló a Cortana en 2015 para integrarla al motor de búsqueda Bing, contando con capacidad para crear recordatorios, responder preguntas y reconocer comandos de voz (Artificial Solutions, 2020).

A la mirada de este recorrido histórico, los chatbots se han integrado cada vez a nuestra vida; principalmente, se les ha reconocido por su integración con motores de búsqueda e entidades empresariales, pero sus atributos pueden ser de utilidad en diversos sectores, tanto económicos como sociales. Según García Brustenga, Fuertes-Alpiste y Molas-Castells (2018), como programas que integran la inteligencia artificial y mantienen la capacidad de simular cierto nivel de diálogo con las personas, los chatbots están manifestándose de manera paulatina en la educación, específicamente en la labor de 
asumir las tareas repetitivas y de nivel cognitivo bajo, aspectos que forman parte del proceso de enseñanza-aprendizaje. Es así como los chatbots, bots conversacionales o asistentes virtuales pueden contribuir con el mejoramiento de la productividad al resolver inquietudes frecuentes e, incluso, asumir un rol de "tutores" en el proceso de aprendizaje.

El propósito de los chatbots en la educación sigue siendo lograr una interacción con base en patrones similares a los que son de uso por parte de los humanos, lo cual se logra mediante los diálogos, ya que son programados para contar con la capacidad de analizar el entorno y proponer soluciones a problemas, interpretando emociones y aportando al máximo en el proceso de enseñanza-aprendizaje. En este sentido, en la relación entre chatbots y educación, se establecen las siguientes precisiones (García Brustenga, Fuertes-Alpiste y Molas-Castells, 2018):

a. La educación es un acto relacional basado en la comunicación y la interacción, por lo tanto, los chatbots cuentan con un potencial educativo que se traduce en el aprovechamiento de su capacidad comunicativa a través del lenguaje natural.

b. De manera similar a otras áreas del conocimiento, los chatbots sirven, en el contexto educativo, para atender las inquietudes de los usuarios 24 horas, los siete días de la semana, con lo cual se evita que los docentes y el personal administrativo respondan inquietudes de índole repetitiva o de respuesta fácil.

c. La introducción de los chatbots en la educación precisa de reflexiones previas por parte de los actores involucrados en el proceso de enseñanza-aprendizaje, ya que los asistentes virtuales nunca reemplazarán el papel del docente en la labor de diseminación del saber. No obstante, pueden ser de inmensa utilidad para resolver dudas de orden administrativo.

d. De igual modo, se puede definir una dinámica complementaria entre el docente y el chatbot, puesto que el profesor puede generar los contenidos de conocimiento a impartir y realizar tutorías personales, mientras que el chatbot puede actuar como asistente en la respuesta de inquietudes sobre algunas temáticas de los cursos de manera permanente.

e. Por otra parte, en el ámbito de la educación y desde la perspectiva de la intencionalidad, se pueden identificar dos tipos de chatbots: uno enfocado en la intencionalidad educativa y el otro en una intencionalidad que no está relacionada directamente con la educación. 
Tamayo y Pérez-Marín (2013) plantean la existencia de chatbots aplicados al sector de la educación, tal es el caso de Laura y Dr. Roland. Laura es un agente que tiene la función de ser una profesora que dialoga con los estudiantes mediante texto, fortaleciendo su proceso de aprendizaje de lengua española. El agente Dr. Roland, por su parte, tiene como misión ayudar a los estudiantes a resolver problemas de matemáticas.

Un ejemplo de chatbot aplicado en el sector específico de la educación superior es el desarrollado recientemente en la Universidad Alicante: Aitana, el cual es capaz de atender dudas sobre el proceso de matrícula de los estudiantes en la aplicación de mensajería instantánea Telegram, donde su nombre identificativo es @UAOficialChatbot; también existe la posibilidad de acceder directamente a través del portal principal de la universidad: www.ua.es. Esto permite contactar el bot tanto desde el navegador como desde la aplicación de Telegram y guardar el contacto para futuras consultas.

Si bien los asistentes virtuales mencionados denotan su relevancia en la educación, fueron pocos los estudios encontrados que hablaran específicamente de los aspectos metodológicos de la construcción de un asistente virtual, así como sus características principales. Tres estudios sobresalientes fueron los de Holtgraves y Han (2007), García Brustenga, Fuertes-Alpiste y Molas-Castells (2018) y Artificial Solutions (2020), los cuales permitieron identificar los principales aspectos a tener en cuenta al momento de desarrollar un chatbot y crear, partir de su análisis las características para el desarrollo de un futuro chatbot para la EIB.

En este sentido es importante tener en cuenta que si bien hay diversidad de referentes, sobre todo en el sector de la educación superior, la descripción de los asuntos metodológicos empleados en el desarrollo y aplicación de un chatbot fue escasa. Resulta importante mencionar, además, que el estudio realizado en esta investigación promueve la realización de estudios previos y pruebas piloto que permitan crear asistentes virtuales coherentes con las necesidades de información de los estudiantes.

Los beneficios del uso de asistentes virtuales en la instrucción se evidencian en incluir colaboración, cooperación, interacción, aprendizaje activo, aprendizaje constructivo y activo, aprendizaje creativo y aprendizaje social: componentes necesarios para ayudar a los estudiantes a prepararse para un futuro que exige de ellos habilidades del siglo XXI. 
Con esta experiencia investigativa se evidencia la oportuna aplicación de la metodología seleccionada (design thinking) a través de la cual se logró alcanzar un estado de empatía con la comunidad académica, al caracterizar el público objetivo, reconocer los actores involucrados en el proceso de educación virtual e identificar las necesidades de información de los estudiantes, imprescindibles al momento de crear una propuesta de asistente virtual en el contexto de la educación superior.

A la luz del rastreo de información que se llevó a cabo por medio de las entrevistas, los grupos focales y las encuestas a diferentes actores involucrados, se lograron identificar los problemas más frecuentes en los procesos comunicativos relacionados, principalmente, con aspectos académicos, administrativos y de Bienestar Universitario, lo cual se explica mediante el desconocimiento, por parte de los estudiantes, de los procesos de educación virtual, el reglamento académico, los procesos administrativos y los canales de comunicación preestablecidos en la institución. Esto confirma la pertinencia del desarrollo de un asistente virtual que permita centralizar las comunicaciones y orientar a los estudiantes.

Con base en el rastreo documental, el análisis del contexto y la identificación de las necesidades de información de los estudiantes, se lograron obtener las características principales del asistente virtual, insumo fundamental para su diseño y aplicación adecuada que logre optimizar los procesos comunicativos entre los estudiantes y quienes coordinan las actividades de modalidad virtual en la EIB.

Luego de llevar a cabo los procesos de rastreo y de exploración sobre los problemas e inquietudes más recurrentes de los estudiantes de Archivística y Bibliotecología de la Escuela Interamericana de Bibliotecología de la Universidad de Antioquia, se logra identificar que la mayoría de las inquietudes presentadas en los foros de la plataforma educativa correspondieron a asuntos académicos que requerían el acompañamiento del profesor encargado de cada curso. En específico, las dudas más comunes son sobre temas académicos (17\%), administrativos $(17 \%)$, y relacionadas con bienestar universitario (14\%).

En este orden de ideas, el presente proyecto es un punto de partida para la creación de un asistente virtual en la Escuela; sin embargo, será necesario llevar a cabo una siguiente etapa de investigación donde se desarrolle el chatbot y se disponga para el uso de los estudiantes. Asimismo, este tipo de iniciativas se constituyen en referentes para generar otras propuestas similares en otras unidades académicas de la Universidad de Antioquia y en otras instituciones de educación superior. 
Esta investigación se ha llevado a cabo gracias al apoyo y respaldo de la comunidad académica de la Escuela Interamericana de Bibliotecología, de su centro de investigaciones CICINF y de la Vicerrectoría de Docencia de la Universidad de Antioquia, dependencia a través de la cual se logró, mediante la convocatoria Con TIC Investigo, la financiación y los recursos necesarios para desarrollarla, así como el acompañamiento y soporte de la unidad de virtualidad: Ude@Educación virtual.

\section{REFERENCIAS}

Almurtadha, Yahya. 2019. "Labeeb: Intelligent Conversational Agent Approach to Enhance Course Teaching and Allied Learning Outcomes Attainment”. Journal of Applied Computer Science \& Mathematics 13 (27): 9-12. https://doi.org/10.4316/jacsm.201901001

Artificial Solutions. 2020. Chatbot: la guía definitiva https://www.artificial-solutions.com/es/chatbots/

Ch'ng, Sue Inn, Lee Seng Yeong y Xin-Yean Ang. 2019. "Preliminary Findings of using Chat-bots as a Course FAQ Tool”, en 2019 IEEE Conference on e-Learning, e-Management \& e-Services (IC3e), 1-5. https://doi.org/10.1109/IC3e47558.2019.8971786

García Brustenga, Guillerm, Marc Fuertes-Alpiste y Núria Molas-Castells. 2018. Briefing paper: los chatbots en educación. Barcelona: Universitat Oberta de Catalunya. https://doi.org/10.7238/elc.chatbots.2018

Griol, David, José Molina, Zoraida Callejas y Ramón López Cózar. 2011. "La plataforma EDUCAGENT: Agentes Conversacionales Inteligentes y Entornos Virtuales aplicados a la Docencia”. Informática Educativa Comunicaciones 13, 15-31. https://e-archivo.uc3m.es/handle/10016/18278

Holtgraves, Thomas y Tai-Lin Han. 2007. "A procedure for studying online conversational processing using a chat bot". Bebavior research methods 39 (1): 156-163. https://doi.org/10.3758/BF03192855

Jo, Sihyeon, Seungryong Yoo, Sangwon Im, Seung Hee Yang, Tong Zuo, Hee-Eun Kim, SangWook Han y Seong-Woo Kim. 2020. "A Scalable Chatbot Platform Leveraging Online Community Posts: A Proof-of-Concept Study”. arXiv. Preprint. https://arxiv.org/abs/2001.03278

Medrano, José, Mario Tejerina y César Castillo. 2019. "Empleo de chatbots educativos como recurso complementario en las prácticas docentes", en SEDICI, 1-5. Buenos Aires: UNLP. http://sedici.unlp.edu.ar/handle/10915/77337

Plattner, Hasso. 2018. Guía del proceso creativo. Miniguía: una introducción al Design thinking + Bootcamp bootleg. Traducido por Felipe González. Institute of Desing at Stanford. https://bit.ly/3eydsHL

Sinha, Sharob, Shyanka Basak, Yajushi Dey y Anupam Mondal. 2020. "An Educational Chatbot for Answering Queries", en Emerging Technology in Modelling and Graphics, 55-60. Singapur: Springer. 
Tamayo, Silvia y Diana Pérez-Marín. 2013. "Análisis de la experiencia de uso de un agente de comprensión lectora con niños en edad escolar". Teoría de la Educación. Educación y Cultura en la Sociedad de la Información 14 (2): 403-429.

https://www.redalyc.org/articulo.oa?id=201028055018

Para citar este texto:

Múnera Torres, María Teresa, Leidy Marisol Salazar Álvarez y Arbey Stiven Osorio Osorio. 2022. "Estudio inicial de un chatbot para estudiantes de la modalidad virtual de la Escuela Interamericana de Bibliotecología”. Investigación Bibliotecológica: archivonomia, bibliotecología e información 36 (90): 13-30.

http://dx.doi.org/10.22201/iibi.24488321xe.2022.90.58452 